\title{
TGRP: A New Hybrid Grid-based Routing Approach for Manets
}

\author{
Hussein Al-Maqbali \\ Department of Information Technology \\ Ministry of Transport and Communications \\ Muscat, Oman
}

Mohamed Ould-Khaoua

Department of Electrical and Computer Engineering

Sultan Qaboos University

Muscat, Oman

\author{
* Khaled Day \\ Department of Computer Science \\ Sultan Qaboos University \\ Muscat, Oman \\ Abderezak Touzene \\ Department of Computer Science \\ Sultan Qaboos University \\ Muscat, Oman
}

\author{
Nasser Alzeidi \\ Department of Computer Science \\ Sultan Qaboos University \\ Muscat, Oman
}

\begin{abstract}
Most existing grid-based routing protocols use reactive mechanisms to build routing paths. In this paper, we propose a new hybrid approach for grid-based routing in MANETs which uses a combination of reactive and proactive mechanisms. The proposed routing approach uses shortest-path trees to build the routing paths between source and destination nodes. We design a new protocol based on this approach called the Tree-based Grid Routing Protocol (TGRP). The main advantage of the new approach is the high routing path stability due to availability of readily constructed alternative paths. Our simulation results show that the stability of the TGRP paths results in a substantially higher performance compared to other protocols in terms of lower end-to-end delay, higher delivery ratio and reduced control overhead.
\end{abstract}

Keywords-MANETs; routing protocols; NS2 simulation; performance evaluation

\section{INTRODUCTION}

A Mobile Ad-hoc Network (MANET) is defined as a collection of autonomous mobile nodes which communicate in the absence of access points. A node in a mobile ad-hoc network works as a host and as a router to serve multi-hop wireless communication and usually has limited power resources. Routing in a mobile ad hoc network is a challenging task since the network's topology changes frequently due to mobility. A node sends control packets to discover destinations and to establish and maintain routes. Since the channel bandwidth and power are often limited, route establishment should be done with minimum control packets and minimum usage of bandwidth and energy.

Many routing protocols have been proposed for MANETs [1] such as topology-based routing protocols (e.g. DSDV [2], AODV [3] and DSR [4] [5]), position-based routing protocols (e.g. Compass [6] and Greedy [7]) and grid-based routing protocol (e.g. GRID [8], EC-GRID [9]). The topology-based MANET routing protocols suffer from low scalability because of the high number of overhead messages and high network latency, especially with high node mobility. The availability of cheap instruments for estimating the position of nodes in a network, like Global Positioning System (GPS) receivers, has motivated many researchers to develop position-based routing protocols for MANETs [10] [11] [12]. Position-based routing protocols can eliminate the need to maintain routes. They use the knowledge of the nodes locations to route packets. Position-based protocols assume that any node is aware of its position, the position of its neighbors as well as the position of the destination. A node can discover its position using a location mechanism such as GPS [13]. It can discover its neighbors' locations by using periodic messages. The nodes use location services to discover destination nodes locations [14].

In position-based routing protocols, each node has an identifier (id) and a current geographic position. Typically in grid-based routing protocols, the physical area is divided into a logical two-dimensional (2D) grid. The logical 2D grid structure allows using cell-by-cell routes where there is a cellhead node in each cell to handle routing. Cell-based routing enhances the scalability of the routing protocol [15]. One node is elected as a cell-head in each grid cell and it has the following responsibilities: (1) forward route discovery requests to its neighbor cells; (2) transmit data packets to neighboring cells; and (3) maintain the routes that pass through its cell.

Each of the three types of existing routing protocols topology-based, position-based and grid-based has limitations. For instance, topology-based protocols generate a large amount of traffic when the network topology changes frequently due to mobility [13]. Position-based protocols suffer from a local minima problem which leads to non-guaranteed message 
delivery [16]. Furthermore, position-based protocols mostly depend on location services [13] such as Home Agent [17] and Grid Location Service [14] to discover geographical locations of destinations. Another limitation of existing grid-based routing protocols is that they use an election approach for selecting cell-head (gateway) nodes which leads to high control packet overhead and high end-to-end delays. In this paper, we propose a new hybrid (proactive and reactive) routing approach which highly utilizes the logical grid environment in order to reduce the number of control packets and increase path stability. The new approach divides the routing in two layers: a proactive layer, where shortest-path trees are constructed and maintained, and a reactive layer, where destination nodes are tracked making use of the constructed shortest-path trees. In the proactive layer information about occupied grid cells is exchanged among nodes. A grid cell is referred to as "occupied" when there is at least one mobile node located in the inner margins of the cell; otherwise it is a "non-occupied" cell. Moreover, if a non-occupied cell becomes occupied, a special control packet (Empty_to_Non-Empty control packet) is flooded (using cell-based flooding) to inform all nodes in the network about this event. In cell-based flooding only one node in each cell (the cell-head) participates in broadcasting the packet to neighboring cell-heads. Similarly when an occupied cell becomes non-occupied, all nodes are informed using a cell-based flooding of a special control packet (NonEmpty_to_Empty control packet). The reactive layer is used to seek for a destination. Any node that wants to establish a connection with an unknown node (not registered in a local Node Table); it starts by sending a Route Request (RREQ) packet to seek for the destination location using cell-based flooding. The proactive layer information is saved in all nodes in the MANET environment. This information enables a cellhead to build a shortest path tree from the cell where it is located to all grid cells. If there is a change in the information about the occupied or non-occupied cells, the tree is reconstructed. The proactive mechanism runs without interrupting the data packets propagation. The reactive layer information (destination nodes location) is also saved at all nodes in a local Nodes Table.

We have conducted an intensive simulation-based performance evaluation of the proposed TGRP protocol and measured the average message delivery ratio, the normalized control overhead and the average end-to-end delay. We have extended the NS2 network simulator, which has been widely used in the literature for studying the performance of MANET routing protocols [15] [18] [19], to evaluate the performance of TGRP and compare it with the performance of GRID protocols. We have studied the performance of TGRP under a variety of network densities and cell sizes. The results show that TGRP outperforms GRID in terms of end-to-end-delays and delivery ratio. Furthermore, TGRP competes well with GRID in terms of control packet overhead. This paper is a revised and expanded version of our previous conference paper presented in [20].

The rest of this paper is organized as follows. Section 2 describes the proposed TGRP structure including its proactive layer and reactive layer mechanisms. Section 3 presents a simulation-based performance evaluation of TGRP compared to grid-based protocols. Section 4 concludes the paper.

\section{The TREE-BASED GRID ROUTING PROTOCOL (TGRP)}

Like other grid-based protocols, TGRP divides the physical area into a logical two-dimensional grid of equal size cells (see Figure 1). In TGRP, a packet travels from a source node to a destination node by hopping from cell to cell making use of a previously constructed shortest path tree to decide at each hop the next cell to go towards the destination cell. A selected cellhead in each cell is responsible of forwarding the packets via that cell. The union of the cell-heads forms a backbone of the MANET.

Each node maintains four tables, namely Neighbors Table, Occupied Cells Table (OCT), Nodes Table and Tree Table (TT). The Neighbors Table in a given node contains lists of neighboring nodes. Each list represents one of the neighboring cells and lists the ids of all nodes in that cell. The Occupied Cells Table contains addresses of all occupied cells (i.e., the non-empty cells) whereas the Tree Table, which is the routing table, contains the occupied cells addresses and the next hop (next cell) info to reach them. The next hops are obtained by building a shortest path tree using an efficient algorithm. The Nodes Table contains the ids of all nodes and their locations (cell addresses). A cell address is a pair of (x,y) coordinates in the logical grid assuming the address of the bottom left corner cell is $(1,1)$.

Figure 1, Figure 2, Table 1 and Table 2 show respectively an example of a MANET environment, an Occupied Cells Table, a shortest path tree created at node 1 and its representation in a routing table (a Tree Table).

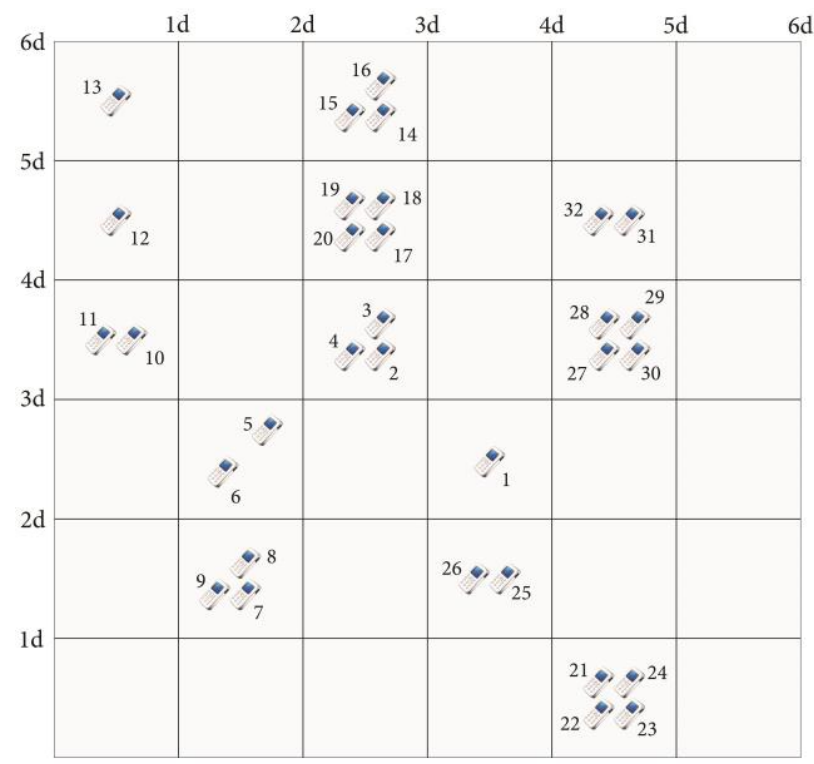

Fig. 1. An example of a grid-based MANET environment

TABLE I. OCCUPIED CELls TABle OF Figure 1

\begin{tabular}{|l|l|l|l|l|l|l|l|l|l|l|l|l|}
\hline$(5,1)$ & $(2,2)$ & $(4,2)$ & $(2,3)$ & $(4,3)$ & $(1,4)$ & $(3,4)$ & $(5,4)$ & $(1,5)$ & $(3,5)$ & $(5,5)$ & $(1,6)$ & $(3,6)$ \\
\hline
\end{tabular}




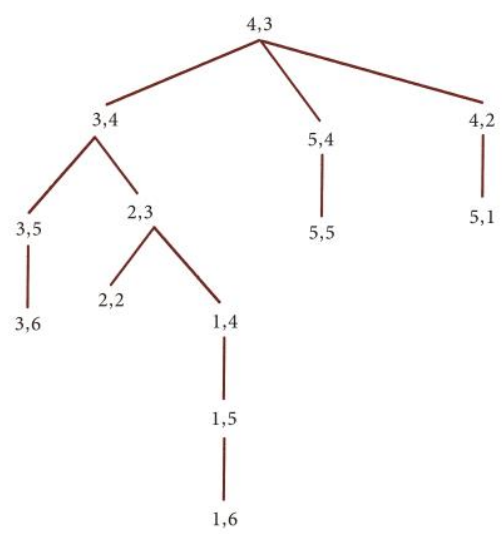

Fig. 2. A shortest path tree at Node 1 of Figure 1

TABLE II. OCCUPIED CELLS TABLE OF FIGURE 1

\begin{tabular}{|l|l|l|l|l|l|l|l|l|l|l|l|l|}
\hline Destination & $(3,4)$ & $(5,4)$ & $(4,2)$ & $(3,5)$ & $(2,3)$ & $(3,6)$ & $(2,2)$ & $(1,4)$ & $(1,5)$ & $(1,6)$ & $(5,5)$ & $(5,1)$ \\
\hline Next Hop & $(3,4)$ & $(5,4)$ & $(4,2)$ & $(3,4)$ & $(3,4)$ & $(3,4)$ & $(3,4)$ & $(3,4)$ & $(3,4)$ & $(3,4)$ & $(5,4)$ & $(4,2)$ \\
\hline
\end{tabular}

\section{A. Control Packets}

TGRP uses control packets to build and maintain its tables. The TGRP proactive layer creates a shortest path tree at each node by using information available in the Occupied Cells Table. Thus, it is important to ensure consistency of the information in the Occupied Cells Table of all nodes in the environment. We do that by using dedicated control packets. These control packets are used to register new occupied cells and to delete empty cells. The following control packets are used for this purpose:

a) Empty_to_Non-Empty (ENE) packet: when a node enters an empty cell, it floods (using cell-based flooding) an Empty_to_Non-Empty (ENE) packet. This packet contains the cell address of the entered cell. The nodes which receive this packet update their OCT tables by adding the cell address of the entered cell.

b) Non-Empty_to_Empty (NEE) packet: when a cellhead leaves a cell and there is no other node left in that cell, it floods (using cell-based flooding) a Non-Empty_to_Empty (NEE) packet. This packet contains the address of the cell that has become empty. Any node recieving this packet updates its OCT table by deleting from it the cell that has become empty.

c) EXIT packet: when any node moves out of its current cell to a neighboring cell, it transmits an EXIT packet to tell the neighbors about its new location.

d) INFO packet: when a cell-head node receives an EXIT packet from a node in its cell, it replies (using unicasting) by sending an INFO packet which contains the Neighbors Table. The sender of the EXIT packet replaces its Neighbors Table with the received one.

The reactive layer uses the following control packets:

e) Route Request (RREQ) packet: A Route Request packet piggy-backs the geographic location (cell address) of the source node to be recorded by all reachable nodes in the
MANET. Every cell-head node will rebroadcast this packet after piggy-backing its geographic location.

f) Destinaion Location (DLOC) packet: when a destination node receives a RREQ packet, it floods (using cellbased flooding) its cell address location using a Destination Location (DLOC) packet to all nodes in the network. Any node receiving this DLOC packet updates its Nodes Table.

\section{B. Building and Maintaining Shortest Path Trees}

At each node a shortest path tree rooted at that node is constructed and used to build a Tree Table which guides the routing of data packets towards their destination cells. The shortest path tree is constructed using the information about occupied cells gathered during the proactive layer of the protocol. The tree is a breadth-first search tree of the graph of occupied cells. The vertices of this graph are the occupied cells and two occupied cells are connected by an edge if they are neighboring cells in the grid. The breadth-first search algorithm is outlined in Figure 3. It uses a FIFO queue data structure storing a list of occupied cells not yet visited in the search. The breadth-first search algorithm starts by enqueueing the root cell in the initially empty queue and then loops dequeueing at each iteration one cell from the queue and enqueueing its unvisited adjacent occupied cells until the queue becomes empty. In each iteration the links between the dequeued cell and enqueued adjacent cells are recorded in the Tree Table as (next hop) links on the shortest path tree.

\section{Build Tree Algorithm:}

Initialize Queue to empty

Initialize Tree Table to empty

Register this node's cell (root cell) in the Tree Table

Enque this node's cell (root cell)

While Queue is not empty

$>$ Dequeue one cell $C$ from Queue

$>$ For each neighboring cell $C^{\prime}$ of $C$ do the following:

○ If $C^{\prime}$ is an empty cell (not listed in the OCT table) then ignore it

- If $C^{\prime}$ is already registered in the routing table then ignore it

○ If $C^{\prime}$ is occupied (listed in OCT) and not registered in the routing table then enqueue $C$, in Queue and register it in the Tree Table ( $C$ is the next hop from $C$ ' towards root)

Fig. 3. Construction of the shortest path tree and tree table.

The time complexity of this construction is $\mathrm{O}(V+E)$ where $V$ is the number of occupied cells and $E$ is the number of (occupied cell, adjacent occupied cell) links. In the worst case, $V$ is equal to the total number of grid cells and $E$ is less than $4 V$ (since each cell has at most 8 adjacent non empty cells). The shortest path tree construction algorithm is therefore $\mathrm{O}(N)$ where $N$ is the number of occupied grid cells.

The shortest path tree has to be rebuilt when there is a change in the occupied/empty status of the cells. To reduce the 
number of times the tree is rebuilt, we use a valid Boolean flag indicating whether the current tree is valid or not depending on changes in the Occupied Cells Table. A node does not rebuild the tree until it has to route a data packet and the valid flag is false.

\section{Cell-Based Flooding}

Any node that decides to initiate a route discovery (if there is no information about the destination neither in the Neighbors Table nor in the Nodes Table) broadcasts a route request (RREQ) to all its adjacent cells. There are two possible cases for a node receiving the RREQ:

Case 1: Cell-Head Node: A cell-head node receiving the RREQ has to flood (cell-based flooding) a route reply (RREQ) packet. In cell-based flooding only one node in each cell, the cell-head, participates in the flooding. If any node receives a previously processed RREQ (detected by checking the node sequence number in its Nodes Table), the node discards it and does not forward it. RREQ piggybacks the location (cell address) of the previous hop node which is used to update the Neighbors Table.

Case 2: Non Cell-Head Node. It broadcasts (using cellbased flooding) a Destination Location (DLOC) packet if it is the destination; otherwise it records the cell location information of the previous forwarding node and then discards the RREQ packet.

Only one node in each cell (the cell-head) participates in rebroadcasting the RREQ. This mechanism is called cell-based flooding (as opposed to total flooding used in AODV for example). Once the source node receives the Destination Location DLOC packet, it starts forwarding data packets to the destination using the shortest path tree next hop links recorded in the Tree Table. If the destination node moves out of its cell to a new one, it should broadcast (using cell-based flooding) a Destination Location DLOC packet. This will not affect the ongoing transmission of data packets to the destination.

\section{Cell-Head Selection in TGRP}

After building the Neighbors Table with the most recent information, the selection of the cell-head node becomes simple and fast. The node with the highest id in a cell is implicitly chosen as the cell-head of that cell without any additional overhead.

All the nodes in the MANET have the same OCT table which leads to avoid using any special packets for maintaining cell-heads. Notice that in the GRID protocol a RETIRE packet is sent by a cell-head when it leaves its cell to another cell. Cell-heads are used in TGRP as a backbone for the cell-based flooding mechanism and there is no need for the RETIRE packet.

The cell-head could be chosen by any mechanism such as selecting the node with highest id as we do here, (or lowest id). To avoid overloading the nodes with high ids (or low ids), a node could create and use a random number as a varying id. In the unlikely case of two equal random numbers drawn at two mobile nodes located in the same cell any of them can be chosen as cell-head of the cell because both nodes have the same OCT table.

\section{E. Operations of the Proactive and Reactive Layers in TGRP}

Any node joining the MANET executes an initialization phase in which it starts by determining its geographic location. It continues monitoring its location periodically until it leaves the MANET.

TGRP is divided into two layers: a proactive layer and a reactive layer; each layer has its own mechanisms. The main function of the proactive layer is to maintain up-to-date the Occupied Cells Table and the Tree Table. All nodes in the MANET environment should have the same copy of the Occupied Cells Table (see Figure 4) which is maintained using the proactive layer control packets. Figure 4 outlines the Occupied Cells Table maintenance mechanisms.

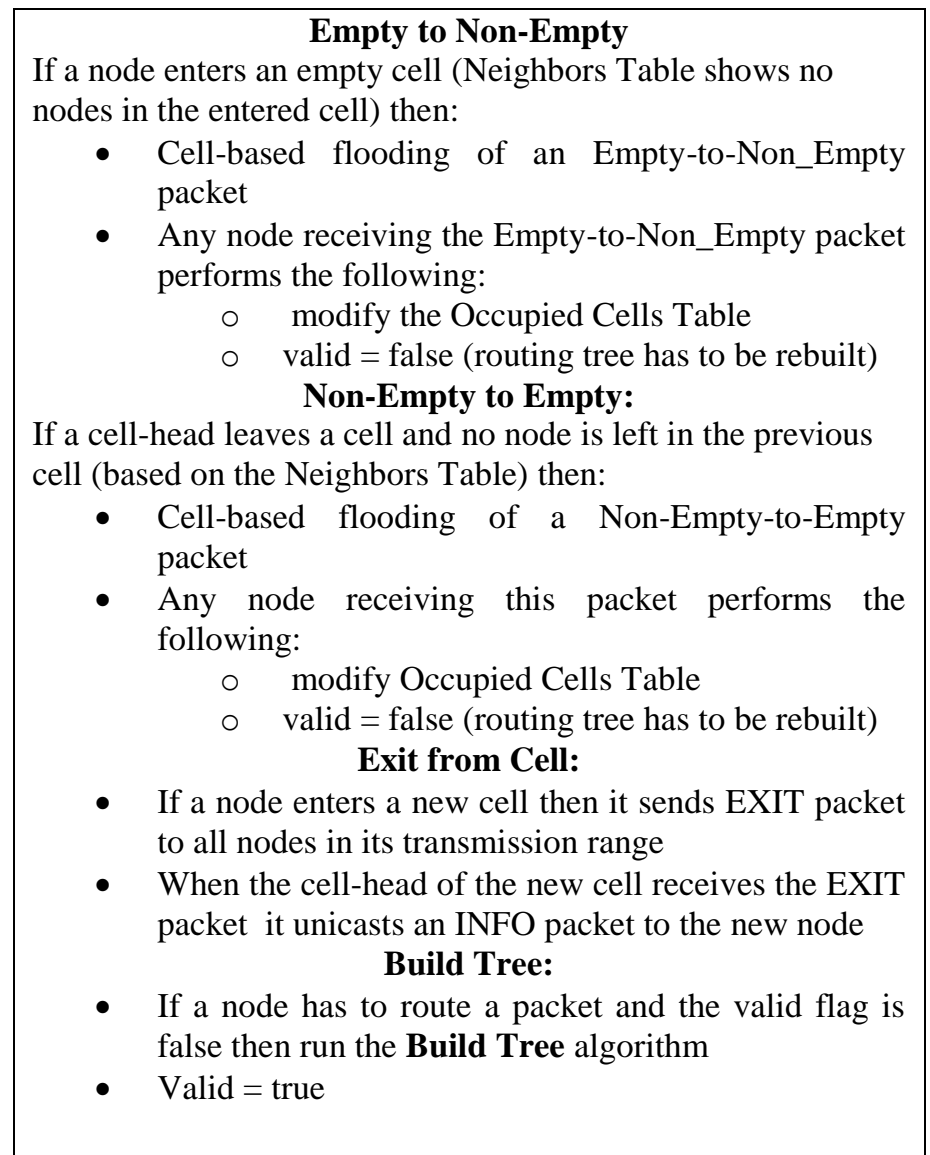

Fig. 4. The operations of the proactive layer in TGRP

The reactive layer is used to track the location of a destination and to ensure this information is known by all nodes in the MANET. Figure 5 outlines the mechanisms of this layer.

\section{Routing of a Received Data Packet}

- If no information about the destination in the Nodes Table then cell-based flooding of RREQ

- If cell-head receives RREQ request (first time) then retransmit RREQ

- If non-cell-head receives RREQ request and it is not the destination node then do nothing 
- If a destination node receives RREQ then cell-based flooding of Destination Location packet

\section{Destination Location}

- If the destination moves to another cell then cellbased flooding of a Destination Location packet

Fig. 5. The operations of the reactive layer in TGRP.

\section{A Simulation-BASEd Performance Evaluation}

In order to investigate the effect of using the hybrid TGRP protocol, we have extended an original implementation of NS2 (version 3.4) with implementations of the protocols GRID and TGRP in order to evaluate and compare their performance. The performance evaluation of TGRP has been conducted using the simulation model and parameters outlined in Table 3. The evaluation study analyzes the impact of the network density and node mobility speed on the three performance metrics packet delivery ratio, normalized control overhead and average end-to-end delay for two different cell sizes.

We use the term cell size to refer to the cell side length. As shown in Table 3, two cell sizes 141 meters and 190 meters will be tested in our experiments to show the impact of cell sizes on TGRP and GRID protocols.

TABLE III. SIMULATION PARAMETERS

\begin{tabular}{|c|c|}
\hline Communication type & CBR \\
\hline CBR sending rate & 4 packets per second \\
\hline Simulation area & $1000 \mathrm{~m} \times 1000 \mathrm{~m}$ \\
\hline Simulation protocols & TGRP, GRID \\
\hline Mobility model & Steady-state random waypoint \\
\hline Number of nodes & $60,100,200,300,400$ \\
\hline Nodes average speed & $2,4,6,8,12,16,20$ (meters/second) \\
\hline Average pause time & 2 (Delta $=1$ seconds) \\
\hline Number of connections & 30 connections \\
\hline Transmission range & 300 meters \\
\hline Physical link bandwidth & 11 Mbps \\
\hline Number of simulation trials & 40 times \\
\hline Simulation time & 1000 seconds \\
\hline Cell side length (cell size) & 141,190 meters \\
\hline
\end{tabular}

\section{A. Impact of Network Density}

This section presents results of studying the impact of network density on the performance of TGRP compared to GRID. The network density has been varied by deploying 60 , $100,200,300$ and 400 mobile nodes in a fixed geographic area of dimensions $1000 \mathrm{~m} \times 1000 \mathrm{~m}$. The nodes in the network move according to the steady-state random waypoint mobility model with average speed of 6 meters per second. The number of connections between randomly selected peer sources and destinations has been fixed to 30 , all established during the simulation time. Each source node in a connection sends four packets per second to the corresponding destination and each packet is of size 512 bytes.

\section{F. Delivery Ratio:}

Figure 8 shows the effect of the network density on the packet delivery ratio of the TGRP and GRID protocols for different cell sizes. It reveals that TGRP with a grid cell size of 141 meters exhibits a better performance compared to GRID in terms of packet delivery. The stability of the path in TGRP allows nodes to keep pumping data packets without interruption. This stability comes from the existence of alternative paths in the OCT table. Anytime there is a change in the OCT table, a new shortest path tree is constructed proactively by the affected nodes without affecting the ongoing communications.

The mobility of a source node does not affect the path. As long as the destination node remains in the same destination cell, the packets can still be routed correctly based on the information in the Node Table. The source node can continue sending packets while moving around in the MANET environment. There is no need to inform all other nodes about its new location when it moves to a different cell. It just sends an EXIT packet to its neighbor nodes and rebuilds its shortest path tree.

The stability of paths also comes from avoiding the use of RETIRE packets and cell-head re-election when cell-heads move between cells. Cell-heads just broadcast EXIT packets when they move to different cells. When a cell-head A moves to a new cell, the cell-head B of that new cell sends a small INFO packet to A containing the list of nodes located in the neighboring cells

The increase of the delivery ratio in TGRP also comes from the fact that cell-heads in GRID protocols discard some data packets when they are not involved in routing packets for any reason such as a change in the path due to the moving of a source node to a different cell or because of received ERROR packets. This is not the case in TGRP because all nodes have the capability to forward packets to destination nodes and all nodes have consistent information about paths to destination nodes.

The simulation results show that TGRP works better with a cell size 141 meters than 190 meters. This is because of the lack of consistency between the transmission range (300 meters) and the cell size 190 meters. For example, with a cell size of 141 meters and a transmission range of 300 meters, the adjacent neighbors of a cell are approximately covered by the transmission range which will allow nodes in this cell to communicate with each other. In the example of Figure 6, a grid size of 141 meters is assumed. In this figure node 20 wants to send a data packet to node 1 through node 4 . Node 20 sends its packets to node 4 . Node 4 discovers from its Neighbors Table that the destination node 1 is reachable in the next hop. Node 4 delivers the packet to the destination node 1 . If however a cell size of 190 meters is assumed, then when node 4 tries to forward the packet received from node 20 to node 1 , the packet is dropped because the transmission range of node 4 does not cover node 1 (see Figure 7). 


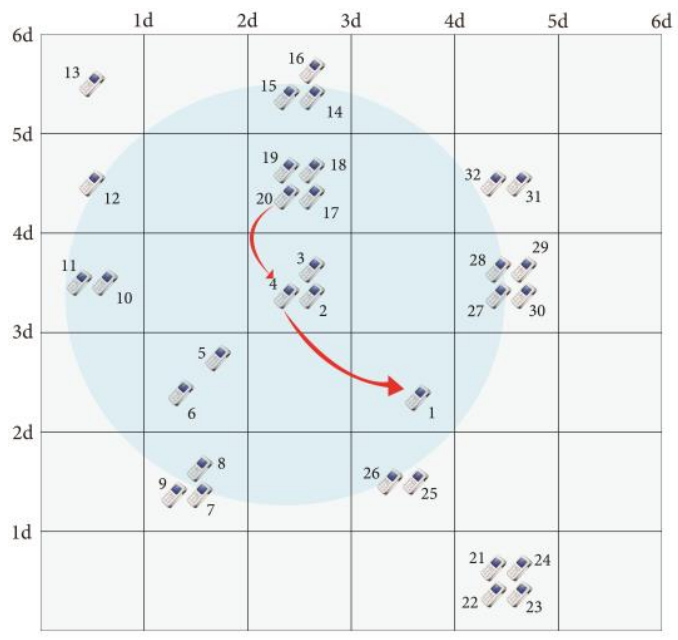

Fig. 6. Grid environment with cell size 141 meters

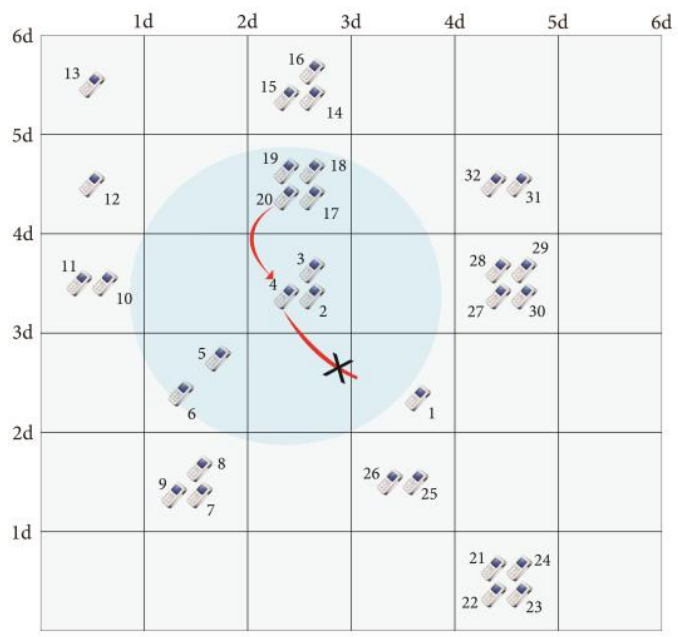

Fig. 7. Grid environment with cell size 190 meters

We observe from the results of Figure 8 that TGRP outperforms GRID protocols in terms of delivery ratio. This can be justified by the fact that the stability of the paths resulting from using the proactive layer information (availability of alternative paths) leads to an increase in the number of delivered packets to their destinations. Figure 8 also shows that TGRP incurs a higher delivery ratio with size 141 meters compared to 190 meters.

Figure 8 shows that sparse environment (density $=60,100$ nodes) has a negative effect on the delivery ratio of TGRP whereas dense environment has a positive effect due to the ability of building paths to destinations when the density is high. Moreover, the delivery ratio with 300 nodes is slightly better than the delivery ratio with 400 nodes due to the fact that 400 nodes broadcast more EXIT packets which affects the propagation of data packets.

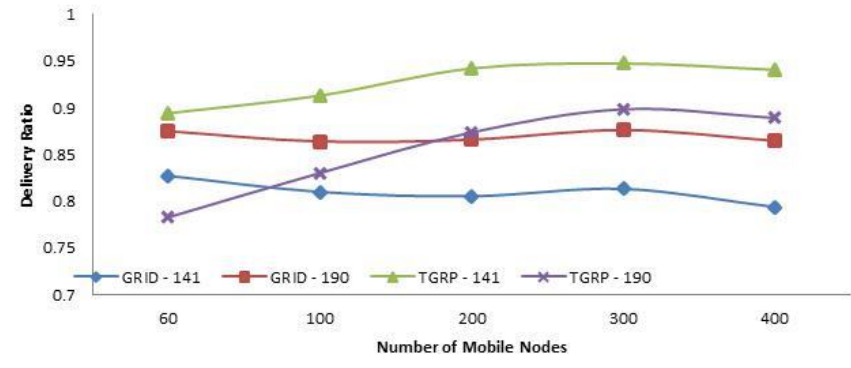

Fig. 8. Delivery ratio vs. number of mobile nodes for TGRP and GRID. Mobility speed $=6 \mathrm{~m} /$ number of connections $=30$, CBR packet rate $=4$ packets/sec.

\section{G. Control Overhead:}

In this section, we present simulation results measuring the normalized control overhead which is defined here as the number of generated control packets per delivered data packet. Figure 9 shows that TGRP is more scalable than GRID in terms of normalized control overhead when varying the number of nodes (and hence the network density). TGRP has exhibited an inverse relationship between network density and normalized control overhead whereas GRID has exhibited a positive relationship.

The proactive layer control packets (Empty-to-Non-Empty and Non-Empty-to-Empty) are affected inversely with the increase of density. Increasing the number of nodes reduces the need to send those packets because those packets are sent when the status of a cell changes from empty to non-empty or vice versa. These changes are reduced with an increased number of nodes in the cells. Thus, in TGRP, the number of control packets needed to deliver data packets is reduced with increased number of nodes.

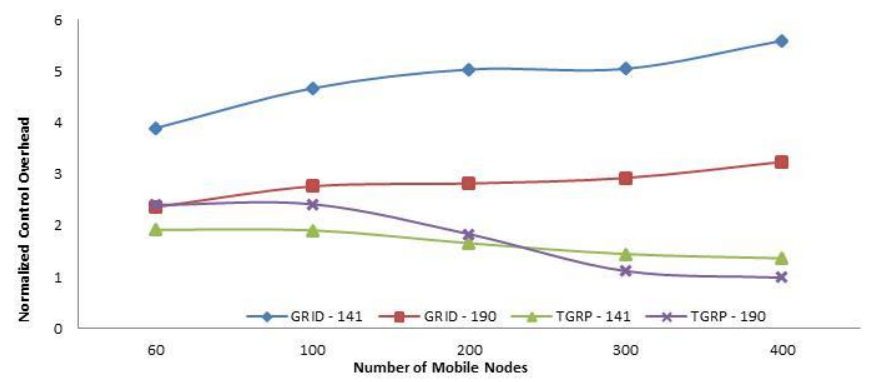

Fig. 9. Normalized control overhead vs. number of mobile nodes for TGRP and GRID. Mobility speed $=6 \mathrm{~m} / \mathrm{s}$, number of connections $=30$, CBR packet rate $=4$ packets/sec.

When the density is less than 200 nodes, TGRP with cell size 190 meters needs more control packets to submit data packets compared to TGRP with cell size 141 meters. This is due to the disconnection problem illustrated in Figure 7. When the density is higher than 200 nodes, the disconnecting of paths is reduced due to the existence of more nodes in cells which leads to reduce the number of control packets. 


\section{H. End-to-End Delay:}

Figure 10 shows that TGRP exhibits much better performance than GRID in terms of end-to-end delay. The TGRP proactive layer provides high path stability which results in a tremendous improvement in the average end-to-end delay compared to GRID (over $80 \%$ improvement compared to GRID).

The mechanism of path maintenance in TGRP does not affect the pumping of data packets. The maintenance of the paths is done in the proactive layer (in the background). There are always chances of existence of alterative paths. All these factors lead to the superiority of TGRP. Figure 10 also reveals that the increase in the density of the network leads to improve the average end-to-end delay for all protocols. In spite of the big difference between protocols, they all exhibit the same behavior.

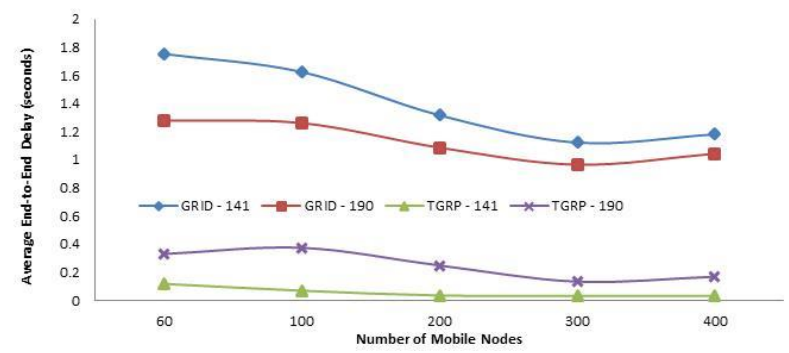

Fig. 10. Average end-to-end delay (seconds) vs. number of mobile nodes for TGRP and GRID. Mobility speed $=6 \mathrm{~m} /$ number of connections $=30, \mathrm{CBR}$ packet rate $=4$ packets $/ \mathrm{sec}$

\section{Impact of Node Mobility}

To study the effect of mobility on TGRP compared to GRID, we set a fixed number of 200 nodes and a fixed number of 30 connections and vary the node mobility speed. The nodes are placed over a network area of $1000 \mathrm{~m} \times 1000 \mathrm{~m}$ using steady-state random waypoint mobility model with a variety of node mobility speeds as shown in Table 4.

TABLE IV. PARAMETERS FOR NODE SPEED

\begin{tabular}{|c|c|c|c|c|c|c|c|}
\hline $\begin{array}{c}\text { Average Node } \\
\text { Speed } \\
\text { (meters/second) }\end{array}$ & 2 & 4 & 6 & 8 & 12 & 16 & 20 \\
\hline $\begin{array}{c}\text { Delta of Node } \\
\text { Speed } \\
\text { (second) }\end{array}$ & 1 & 2 & 4 & 6 & 10 & 12 & 18 \\
\hline
\end{tabular}

\section{J. Delivery Ratio:}

Figure 11 reveals that TGRP with cell size 141 meters is more scalable and stable in terms of delivery ratio when varying the node mobility speed. The delivery ratio of TGRP is not affected by the increase of the node mobility speed. There is no election mechanism that effects the propagation of data packets. TGRP with cell size 190 meters has the same behavior as TGRP with cell size 141meters but it has lower delivery ratio because of the disconnection problem illustrated in Figure 7.

TGRP depends on its proactive layer control packets to ensure consistency of the Occupied Cells Table and hence the stability of the routing paths. With a high number of mobile nodes (high density), the number of control packets remains approximately the same when increasing the speed. A path to destination is not broken by the moving of nodes between cells. As long as the empty/non-empty status of the cells is not affected, the routing paths (cell-based paths obtained from the shortest-path tree) remain valid. There are only two control packets which are affected by increasing the mobility speed of the nodes: Destination Location packet and EXIT packet. The proactive layer increases the stability of the connected paths which leads to reduced effect of the mobility speed on the delivery ratio.

GRID with cell size 190 meters works better than with cell size 141 meters. The paths are constructed with less number of hops and less number of RETIRE packets.

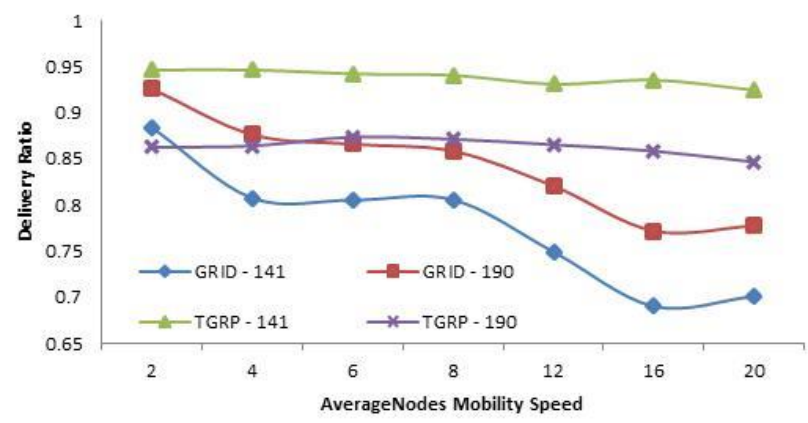

Fig. 11. Delivery ratio (\%) vs. averagenode speed for TGRP and GRID. Number of CBR connections $=30$, number of nodes $=200$, CBR pacekt rate $=4$ packets $/ \mathrm{sec}$.

Overall, TGRP shows high scalability in terms of delivery ratio compared to GRID when increasing the node mobility speed. It shows improvement by at least $10 \%$ compared to GRID. It is slightly affected by the increasing of mobility speed. The scalability comes from the stability of the cellbased paths in the TGRP protocol. The paths are cell-based paths and not node-based paths.

\section{K. Control Overhead:}

Figure 12 shows that TGRP has consumed $40 \%$ less control packets than GRID to deliver data packets. There are only three types of control packets which are needed more with higher node mobility: Destination Location packets, EXIT packet and INFO packet. The number of Empty-to-Non-Empty packets and Non-Empty-to-Empty packets is affected by the number of nodes in the environment but not much by the mobility speed. If there are enough mobile nodes, there is little need for these control packets since changes in the empty/non-empty status of cells are unlikely to take place. If however the number of nodes is small then higher mobility causes more empty/non-empty status changes and hence the number of status change control packets can be large.

TGRP has used less control packets to deliver data packets compared to GRID when the average mobility speed is less than 8 meters per second. TGRP with cell size 141 meters needs about one to two control packets to deliver one data packet whereas it needs more than 2 control packets to deliver one data packet when the mobility speed exceeds 8 meters per 
second. TGRP with cell size 190 meters uses approximately the same number of control packets with different mobility speeds to deliver one data packet. That is because, a grid with cell size 141 meters has more cells than a grid with cell size 190 meters which leads to have more Empty-to-Non-Eempty and Non-Empty-to-Empty packets.

Figure 12 also reveals that GRID is affected negatively by increasing the mobility speed in terms of normalized overhead. Increasing the node mobility speed leads to increasing the number of broken connections and therefore the number of control packets used to reestablish the broken connections.

Overall, TGRP is more stable than GRID in terms of control overhead when the mobility is increased.

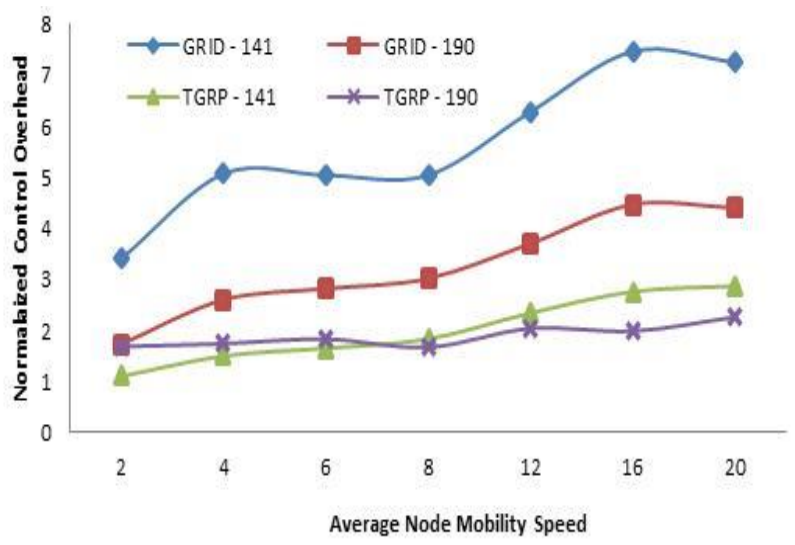

Fig. 12. Normalized control overhead vs. Average Node Mobility Speed for TGRP and GRID. Number of CBR Connections $=30$, number of nodes $=200$, CBR packet rate $=4$ packets $/$ sec .

\section{End-to-End Delay:}

Figure 13 shows that TGRP with cell size 141 meters outperforms by far GRID in terms of end-to-end delay for different node mobility speeds. It shows that it is a very stable and scalable protocol in terms of end-to-end delay. It shows an improvement of about $90 \%$ compared to GRID.

The availability of alternative paths (provided by recalculating the shortest path trees in the proactive layer) leads to have very stable connections. In addition to that, the paths (cell-based paths) are not affected by node mobility as long as the cells forming the paths remain occupied. Figure 13 also reveals that increasing the mobility speed affects negatively GRID in terms of end-to-end delay. It leads to have broken connections which requires in GRID to re-establish the connections using more control packets.

At low mobility speed, TGRP with cell size 141 meters has the lowest average delay among the evaluated protocols. Overall, Figure 13 also shows the effect of node mobility on the average end-to-end delay for the GRID protocol.

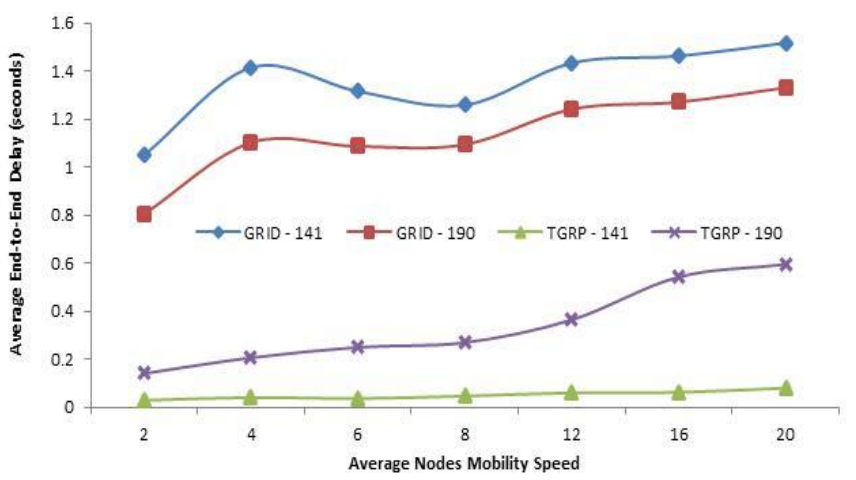

Fig. 13. Average end-to-end delay vs. average node speed for TGRP and GRID. Number of CBR connections $=30$, number of nodes $=200, \mathrm{CBR}$ packet rate $=4$ packets $/$ sec.

\section{CONCLUSION}

We have proposed and evaluated the performance of a new routing protocol called Tree-based Grid Routing Protocol (TGRP), which uses a new hybrid proactive and reactive routing approach in grid-based MANETs. In TGRP, there is a proactive layer which builds and maintains a table called Occupied Cells Table and builds from it shortest path trees between occupied cells. There is also a reactive layer in TGRP that is responsible for discovering the location of destination nodes by exploiting the constructed shortest path trees. The performance of the proposed TGRP protocol has also been studied and compared with the performance of GRID using extensive simulation experiments. The performance has been evaluated in terms of end-to-end delay, delivery ratio and control overhead for a variety of network density and node mobility conditions with two different cell sizes. The results have shown that TGRP scales better than GRID in terms of delivery ratio and control overhead and it is by far superior to GRID in terms of end-to-end delay.

\section{REFERENCES}

[1] H. Walia, E. M. Singh and D. R. Malhotra, "A Review: Mobile Ad Hoc Routing Protocols", International Journal of Future Generation Communication and Networking vol. 9, no. 2 (2016), pp. 193-198.

[2] C. E. Perkins and P. Bhagwat, "Highly Dynamic Destination-Sequenced Distance-Vector Routing (DSDV) for Mobile Computers", Symposium on Comm. Architectures and Protocols, 1994.

[3] C. E. Perkins and E. M. Roycr, "Ad hoc on demand distance vector (AODV) routing (internet-draft)", Mobile Ad-hoc Netwrok (MANET) Working Group, IETF, 1998.

[4] J. Broch, D. B. Johnson and D. A. Maltz, "The dynamic source routing protocol for mobile ad hoc networks (Internet-draft)", Mobile Ad-hoc Network (MANET) Working group, 1998.

[5] D.B. Johnson and D.A. Maltz, "Dynamic Source Routing in Ad hoc Wireless Networks", Mobile Computing, Kluwer Academic Publishers, pp. 153-181, 1996.

[6] E. Kranakis, H. Singh and . J. Urrutia, "Compass routing on geometric networks", 11th Canadian Conference on Computational Geometry (CCCG'99), 1999. 
[7] G. G. Finn, "Routing and addressing problems in large metropolitanscale internetworks", Technical Report ISU/RR-87-180, USC ISI, Marina del Ray, CA, 1987.

[8] W.-h. Liao, J.-p. Sheu and Y.-c. Tseng, "Grid: A Fully Location-Aware Routing Protocol for Mobile Ad Hoc Networks", Telecommunication Systems, vol. 18, pp. 37-60, 2001.

[9] C.-M. Chao , J.-P. Sheu and C.-T. Hu, "Energy-Concerving Grid Routing Protocol in Mobile Ad Hoc Networks," in Proc. of IEEE 2003 Int'l Conference on Parallel Processing, 2003.

[10] Shahab Kamali, Jaroslav Opatrny, "A postition Based Ant Colony Routing Algorithm for Mobile Ad-hoc Netwroks", Academy Publisher, Journal of Networks, pp. vol. 3, no. 4, 2008.

[11] G. Finn, "Routing and addressing problems in large metropolitan-scale internetworks", Technical Report, University of Southern California, 1987.

[12] E. Kranakis, H. Singh, and J. Urrutia, "Compass routing on geometric networks", in 11th Canadian Conference on Computational Geometry (CCCG'99), 1999.

[13] A.E. Abdallaha, T. Fevensa, J. Opatrnya, and I. Stojmenovic, "Poweraware semi-beaconless 3D georouting algorithms using adjustable transmission ranges for wireless ad hoc and sensor networks", Ad-Hoc Networks, vol. 8, no. 1, pp. 15-29, January 2010.
[14] K. Omer and D. Lobiyal, "Performance Evaluation of Location Update Schemes for MANET", The International Arab Journal of Information Technology, vol. 6, no. 3, 2009.

[15] W.-H. Liao, Y.-C. Tseng, J.-P. Sheu, "Grid: A Fully Location-Aware Routing Protocol for Mobile Ad Hoc Networks", Telecommunication Systems, vol. 18, pp. 37-60, 2001.

[16] A.E. Abdallah, T. Fevens, J. Opatrny, "High delivery rate position-based routing algorithms for 3D ad hoc networks", Computer Communications , vol. 31, no. 4, 2008.

[17] Stojmenovic I., "Home Agent Based Location Update and Destination Search Schemes in Ad Hoc Wireless Networks", Advances in Information Science and Soft Computing, 2002.

[18] S. Kurkowski, T. Camp, and M. Colagrosso, "MANET simulation studies: the incredibles", ACM SIGMOBILE Mobile Computing and Communications Review, vol. 9, pp. 50-56, 2005.

[19] M. Bani-Yassein, M. Ould-Khaoua, L. M. Mackenzei, and S. Papanastasiou, "Performance Analysis of Adjusted Probabilistic Broadcasting in Mobile Ad Hoc Networks", International Journal of Wireless Information Networks, vol. 13, pp. 127-140, April 2006.

[20] H. Almaqbali, K. Day, M. Ould-Khaoua, A. Touzene, N. Alzeidi, "A New Hybrid Grid-Based Routing Approach for MANETs", Proc. of CETC2013 Conference on Electronics, Telecommunications and Computers, 5-6 December 2013, Lisbon, Portugal, pp. 81-89. 